\title{
A consistent truncation of IIB supergravity on manifolds admitting a Sasaki-Einstein structure
}

\author{
Kostas Skenderis, ${ }^{a, b}$ Marika Taylor $^{a}$ and Dimitrios Tsimpis ${ }^{c}$ \\ ${ }^{a}$ Institute of Theoretical Physics, \\ Valckenierstraat 65, 1018 XE Amsterdam, The Netherlands \\ ${ }^{b}$ Korteweg-de Vries Institute for Mathematics, \\ Science Park 904, 1098 XH Amsterdam, The Netherlands \\ ${ }^{c}$ Arnold-Sommerfeld-Center for Theoretical Physics, \\ Department für Physik, Ludwig-Maximilians-Universität München, \\ Theresienstraße 37, 80333 München, Germany \\ E-mail: k.skenderis@uva.nl, m.taylor@uva.nl, dimitrios.tsimpis@lmu.de
}

ABSTRACT: We present a consistent truncation of IIB supergravity on manifolds admitting a Sasaki-Einstein structure, which keeps the metric and five real scalar fields. This theory can be further truncated to a constrained one-parameter family that depends on only the metric and one scalar, as well as to a theory with a metric and three scalars. The reduced theory admits supersymmetric and non-supersymmetric $A d S_{5}$ and $A d S_{4} \times \mathbb{R}$ solutions. We analyze the spectrum around the AdS critical points and identify the dual operators.

KeYwords: Flux compactifications, AdS-CFT Correspondence

ARXIV EPRINT: 1003.5657 


\section{Contents}

1 Introduction 1

2 The five-dimensional effective action $\quad 3$

2.1 A three-scalar truncation 4

3 A one-parameter family of constrained Lagrangians 5

3.1 Supersymmetric $A d S_{5} \times S E_{5} \quad 6$

3.2 Non-supersymmetric $A d S_{5} \times \widetilde{S E}_{5} \quad 7$

3.3 Supersymmetric $A d S_{4} \times \mathbb{R} \times \widetilde{S E}_{5} \quad 7$

3.4 Non-supersymmetric $A d S_{4} \times \mathbb{R} \times S E_{5}$

4 Masses $\quad 9$

4.1 The supersymmetric $A d S_{5} \times \mathcal{M}_{5} \quad 10$

4.2 The non-supersymmetric $A d S_{5} \times \mathcal{M}_{5}$

5 Conclusions $\quad 12$

$\begin{array}{ll}\text { A Five-dimensional Sasaki-Einstein manifolds } & 13\end{array}$

$\begin{array}{ll}\text { B Consistent truncation } & 14\end{array}$

\section{Introduction}

Consistent truncation of supergravity theories on compact manifolds has proven to be a useful tool in holography. The truncation reduces the ten-dimensional equations of motion to simpler five-dimensional equations, which in turn makes it easier to find explicit solutions and facilitates the construction of a precise holographic dictionary by making tractable the systematic analysis of the asymptotic structure of the solutions. Moreover, explicit reduction formulae can be used to uplift all results to ten dimensions and discuss their physical content both from the five- and ten-dimensional perspective.

Consistent truncation is not a prerequisite for discussing the system from a fivedimensional perspective and performing a holographic analysis. This can be done without consistent truncation, using the framework of Kaluza-Klein holography [1] which organizes the reduction of all modes according to the way they contribute to the 1-point functions of dual operators: in the computation of the 1-point function of an operator of dimension $\Delta$ only a finite number of fields can contribute, namely the ones that are dual to operators with dimension less than $\Delta$. Thus, effectively one deals with finite number of fields (whose number depends on the dimension of the operator under consideration). In contrast, in consistent truncation one keeps a finite number of fields from the outset. The fact that 
this can be done implies that in the dual theory there is a subset of operators that close under OPEs (at least, in the large $N$, strong coupling limit).

Consistent truncations of maximal supergravities over spheres have been studied extensively [2-6], and it is generally believed that the five-dimensional $\mathcal{N}=8$ supergravity is a consistent truncation of ten-dimensional IIB supergravity on the five-sphere. Spheres are rather special cases of Sasaki-Einstein manifolds, and one of the motivations of the present work was to furnish consistent truncations of IIB on more general five-dimensional Sasaki-Einstein spaces. More recently, two consistent non-supersymmetric truncations of IIB supergravity on five-dimensional Sasaki-Einstein manifolds were presented in [7]; we will return to the precise relation of our results to those of [7] in the following. For other consistent truncations see [8].

Another motivation of the present work was to understand holography for the recently found solutions of [9]. These are solutions of the form $A d S_{4} \times \mathbb{R} \times \mathcal{M}_{5}$, where $\mathcal{M}_{5}$ is a squashed Sasaki-Einstein five-dimensional manifold. These solutions were constructed by exploiting a general geometrical property of all such manifolds, namely their local SU(2) structure, and are therefore independent of the details of $\mathcal{M}_{5}$. In particular, $\mathcal{M}_{5}$ need not be a five-sphere, but can be any five-dimensional manifold admitting a Sasaki-Einstein structure.

It is natural to ask whether the solutions of [9] can be understood within the framework of an effective theory in five dimensions, as is the case for the non-supersymmetric $A d S_{4} \times$ $\mathbb{R} \times S E_{5}$ solution of [10]. ${ }^{1}$ Given the observations of the preceding paragraph, such an effective theory should be a consistent truncation of IIB on $\mathcal{M}_{5}$, where $\mathcal{M}_{5}$ is any fivedimensional manifold admitting a local $\mathrm{SU}(2)$ structure of Sasaki-Einstein type. As we will see in the following, the solutions of both [9] and [10], the supersymmetric $A_{d} S_{5} \times S E_{5}$ solutions, as well as the non-supersymmetric $A d S_{5} \times \widetilde{S E}_{5}$ solutions of [11] (where $\widetilde{S E}_{5}$ is squashed Sasaki-Einstein) can indeed all be recovered as solutions of a five-dimensional consistent truncation of IIB supergravity.

The consistent truncation mentioned in the preceding paragraph is the main result of the present paper, and is presented in eq. (2.8) below. It is a five-dimensional nonsupersymmetric (bosonic) theory consisting of gravity coupled to five real scalars. Moreover, it is possible to show that the theory admits two different further truncations. On the one hand, it reduces to the gravity-scalar sector of the three-scalar truncation of [7] upon suitably eliminating two of the five scalars. From the ten-dimensional point of view, this operation corresponds to setting the three-form flux to zero. On the other hand, the theory can be truncated to a constrained Lagrangian containing the graviton and one real scalar.

Our effective five-dimensional theory admits two $A d S_{5}$ critical points, one supersymmetric and one non-supersymmetric. These uplift to IIB vacua of the form $A d S_{5} \times \mathcal{M}_{5}$, where $\mathcal{M}_{5}$ is a round, squashed $S E_{5}$ in the supersymmetric, non-supersymmetric case respectively. Specializing to the case where the $S E_{5}$ is the five-sphere allows us to unambiguously identify the dual field-theory operators, at least in the supersymmetric case,

\footnotetext{
${ }^{1}$ It is straightforward to generalize the solution of [10] so that the five-dimensional internal manifold is a general Sasaki-Einstein space.
} 
using the well-established AdS/CFT dictionary. However, the fact that none of the fields in our truncation corresponds to higher harmonics on $\mathcal{M}_{5}$ suggests that our analysis may have more general validity.

The remainder of the paper is organized as follows. The Lagrangian of the fivedimensional consistent truncation is presented in section 2. The three-scalar sector of [7] is recovered in section 2.1 as a further truncation. The truncation to the constrained single-scalar theory is described in section 3 . The four classes of supersymmetric and nonsupersymmetric $A d S_{5}$ and $A d S_{4} \times \mathbb{R}$ solutions are presented in sections 3.1 to 3.4. The masses of the fluctuations around the two critical $A d S_{5}$ points and the spectrum of dual operators are discussed in section 4 . We conclude with a discussion of future directions in section 5. Appendix A contains some relevant facts about five-dimensional Sasaki-Einstein geometry, while appendix B contains the technical details of the consistent truncation.

\section{The five-dimensional effective action}

We will now present a consistent truncation of the ten-dimensional IIB supergravity to a five-dimensional effective action containing the graviton and five real scalars. In order to avoid making this section overly technical we will limit ourselves to a schematic description of the reduction ansatz, deferring all technical details to appendix B.

The reduction ansatz for the $10 \mathrm{D}$ metric in the Einstein frame reads:

$$
d s_{10}^{2}=g_{\mu \nu} d x^{\mu} d x^{\nu}+d s^{2}\left(\mathcal{M}_{5}\right),
$$

where $(\mu, \nu)$ are indices along the $5 \mathrm{D}$ spacetime, and $\mathcal{M}_{5}$ is the five-dimensional internal manifold. The latter can be obtained from an associated 'unit' Sasaki-Einstein metric $d s_{S E}^{2}\left(\mathcal{M}_{5}\right)$ through a certain deformation. This metric deformation is parameterized by two real scalars $\psi, \omega$, and corresponds to an overall warping together with a squashing (also sometimes called 'stretching' - depending on one's outlook) of the $\Upsilon(1)$ fiber of the Sasaki-Einstein space. Schematically we have:

$$
d s^{2}\left(\mathcal{M}_{5}\right) \sim e^{2 \psi(x)} d s_{K E}^{2}+e^{2 \omega(x)} u \otimes u,
$$

where

$$
d s_{K E}^{2}+u \otimes u,
$$

is the metric of a canonically normalized 'unit' five-dimensional Sasaki-Einstein space ( $c f$. appendix A for the relevant facts about five-dimensional Sasaki-Einstein manifolds). The precise expression for the internal metric is given in eq. (B.3). The two scalars $\psi, \omega$ are assumed to depend only on the $5 \mathrm{D}$ spacetime coordinates $x^{\mu}$.

The dilaton $\phi$ is assumed to be an independent $5 \mathrm{D}$ spacetime scalar. In addition the ansatz includes two more real 5D spacetime scalars, for a total of five real scalars: a scalar $\chi$ which parameterizes the NSNS three-form flux

$$
H \sim \operatorname{Re} \Omega \wedge d \chi+\chi J \wedge u
$$


and a scalar $\varphi$ which enters in the ansatz for the RR three-form flux

$$
F_{3} \sim J \wedge d \varphi+\varphi \operatorname{Re} \Omega \wedge u .
$$

The real two-form $J$, the complex two-form $\Omega$ and the real one-form $u$ are related to the underlying Sasaki-Einstein structure of the internal manifold. Moreover, the RR one-form flux is assumed to vanish,

$$
F_{1}=0,
$$

while the RR five-form flux depends, in addition to the dilaton, on both $\chi, \varphi$; it consists of two components: one which is proportional to the volume of $\mathcal{M}_{5}$,

$$
F_{5} \sim J \wedge J \wedge u
$$

and another along the 5D spacetime directions which is obtained from the above by tendimensional Hodge-dualization, so that the total five-form flux is self-dual. The precise expressions of the ansätze for the fluxes are given in eqs. (B.7), (B.10).

As shown in detail in appendix $\mathrm{B}$, this reduction ansatz leads to a consistent truncation of ten-dimensional IIB supergravity. The resulting five-dimensional effective action is given by:

$$
\begin{aligned}
\mathcal{L}_{\text {eff }}= & e^{4 \psi+\omega}\left\{R^{(5)}+12(\partial \psi)^{2}+8 \partial \psi \cdot \partial \omega-\frac{1}{2}(\partial \phi)^{2}\right\} \\
& -\frac{1}{5} e^{\omega}\left\{e^{\phi}(\partial \varphi)^{2}+e^{-\phi}(\partial \chi)^{2}\right\} \\
& -2 W^{2}\left(2-\frac{1}{2} \chi \varphi\right)^{2} e^{-4 \psi-\omega}+20 W^{2}\left(1-\frac{e^{2 \omega}}{5 e^{2 \psi}}\right) e^{2 \psi+\omega} \\
& -\frac{5}{4} W^{2} e^{-\omega}\left(e^{\phi} \varphi^{2}+e^{-\phi} \chi^{2}\right)
\end{aligned}
$$

where $W$ is a real constant setting the overall size of the internal manifold. It can be reabsorbed in $\omega, \psi$ without loss of generality.

\subsection{A three-scalar truncation}

The effective action (2.8) can be further consistently truncated to three scalars, in the limit where the scalars $\varphi, \chi$ are eliminated:

$$
\varphi, \chi \rightarrow 0
$$

As can be seen from eqs. (B.7), (B.10), this corresponds from the ten-dimensional point of view to setting the three-form flux to zero. It can be easily verified that this limit is consistent with the $\varphi, \chi$ equations of motion (B.18), (B.19).

In [7], a consistent non-supersymmetric IIB truncation was presented which corresponds to vanishing ten-dimensional three-form flux and whose scalar sector contains precisely three scalars. It can be seen that the further truncation (2.9) of the effective action (2.8) is none other than the gravity-scalar sector of the $\left(m^{2}=8\right)$ consistent truncation of [7]. 
Indeed, this can be explicitly seen as follows. Starting with the effective action (2.8) we can pass to the Einstein frame by setting:

$$
g_{\mu \nu}=e^{-\frac{2}{3}(4 \psi+\omega)} g_{\mu \nu}^{E} .
$$

Moreover, we can diagonalize the resulting kinetic terms by setting:

$$
\psi=\frac{1}{4}(2 u-3 v) ; \quad \omega=-\frac{1}{4}(8 u-3 v),
$$

upon which the effective action reads:

$$
\mathcal{L}_{\text {eff }}=R_{E}^{(5)}-5(\partial u)^{2}-\frac{15}{2}(\partial v)^{2}-\frac{1}{2}(\partial \phi)^{2}+W^{2}\left\{-8 e^{-10 v}+20 e^{-u-4 v}-4 e^{-6 u-4 v}\right\}
$$

The above can be made to match precisely with the gravity-scalar sector of eq. (4.10) of ref. [7] upon performing the constant shifts:

$$
u \rightarrow u+\frac{1}{5} \log \frac{6}{5} ; \quad v \rightarrow v+\frac{1}{5} \log \frac{6}{5},
$$

and setting $W=6 / 5$.

\section{A one-parameter family of constrained Lagrangians}

The effective Lagrangian (2.8) admits a one-parameter family of consistent truncations to a single scalar, which we now describe in detail.

The truncation is obtained by setting:

$$
\begin{aligned}
& \varphi=\alpha e^{-\phi / 2} \\
& \chi=\alpha e^{\phi / 2}
\end{aligned}
$$

and

$$
\alpha, \psi, \omega=\text { constant . }
$$

With the above assumptions the dilaton equation (B.14) reduces to:

$$
\nabla^{2} \phi=0
$$

while the three-form equations (B.18), (B.19) both reduce to:

$$
\alpha\left\{(\partial \phi)^{2}-20 W^{2} x^{-1}\left(\left(2-\frac{1}{2} \alpha^{2}\right) y^{-2}-\frac{5}{4}\right)\right\}=0,
$$

where we set $y:=e^{2 \psi}, x:=e^{2 \omega}$. The internal part of Einstein equations (B.15), (B.16) reduces to:

$$
\begin{aligned}
2 x^{2}+5 x y & =2\left(2-\frac{1}{2} \alpha^{2}\right)^{2} y^{-2}+\frac{5}{2} \alpha^{2} \\
8 x^{2} & =\left(2-\frac{1}{2} \alpha^{2}\right)\left(2-\alpha^{2}\right) y^{-2}+5 \alpha^{2} .
\end{aligned}
$$


The system of equations (3.5) determines $x$ (equiv. $\omega$ ), $y$ (equiv. $\psi$ ) in terms of the free parameter $\alpha$. Finally, the external Einstein equations reduce to:

$$
R_{\mu \nu}=\frac{1}{2}\left(1+\frac{\alpha^{2}}{5 y^{2}}\right) \partial_{\mu} \phi \partial_{\nu} \phi-\frac{1}{3} g_{\mu \nu} \mu(\alpha),
$$

where we defined

$$
\mu(\alpha):=\frac{20 W^{2}}{y}\left(1-\frac{x}{5 y}\right)-\frac{2 W^{2}}{x y^{4}}\left(2-\frac{1}{2} \alpha^{2}\right)^{2}-\frac{5 W^{2}}{2 x y^{2}} \alpha^{2} .
$$

The equations above can be integrated to an effective action

$$
\mathcal{L}_{\text {eff }}^{\prime}=R^{(5)}-\frac{1}{2}\left(1+\frac{\alpha^{2}}{5 y^{2}}\right)(\partial \phi)^{2}+\mu(\alpha),
$$

subject to the constraint (3.4).

The system (3.4), (3.8) admits a number of supersymmetric and non-supersymmetric solutions for different values of the free constant parameter $\alpha$, to which we turn in the following.

\subsection{Supersymmetric $A d S_{5} \times S E_{5}$}

The supersymmetric $A d S_{5} \times S E_{5}$ solution (with maximal supersymmetry in the case where $S E_{5}$ is an $S^{5}$ ) can be obtained from (3.8), (3.4) by setting the dilaton $\phi$ to a constant:

$$
\phi=\phi_{0},
$$

and in addition taking the limit

$$
\alpha \rightarrow 0 \text {. }
$$

Eqs. (3.3), (3.4) are then automatically satisfied, while (3.1) implies:

$$
\varphi=\chi=0 \text {. }
$$

The system (3.5) is then solved for

$$
c_{1} e^{2 \psi}=c_{2} e^{2 \omega}=\frac{1}{\lambda^{2}},
$$

where we have set:

$$
\lambda^{2}:=\left(\frac{5}{6}\right)^{\frac{3}{2}} W^{2} .
$$

From the ten-dimensional point of view, eqs. (3.12), (B.3) imply that the internal space is Sasaki-Einstein:

$$
d s^{2}\left(\mathcal{M}_{5}\right)=\frac{1}{\lambda^{2}}\left(d s_{K E}^{2}+u \otimes u\right),
$$

cf. appendix A. Moreover, as can be seen from (B.7), (B.10), eq. (3.11) sets all fluxes to zero except for the RR five-form:

$$
F_{5}=4 \lambda \operatorname{vol}_{5},
$$

where $v l_{5}$ denotes the volume of the internal space. Finally, the Einstein eqs. (3.6) reduce to:

$$
R_{\mu \nu}=-4 \lambda^{2} g_{\mu \nu}
$$

i.e. the external space is $A d S_{5}$ with inverse radius $\lambda$. 


\subsection{Non-supersymmetric $A d S_{5} \times \widetilde{S E}_{5}$}

A non-supersymmetric $A d S_{5} \times \mathcal{M}_{5}$ solution can be obtained from (3.8), (3.4) by setting the dilaton $\phi$ to a constant:

$$
\phi=\phi_{0},
$$

and in addition setting:

$$
\alpha^{2}=2 ; \quad e^{2 \psi}=\frac{2}{\sqrt{5}} ; \quad e^{2 \omega}=\frac{\sqrt{5}}{2} .
$$

As is straightforward to verify, the above assignments solve eqs. (3.3), (3.4), (3.5). From the ten-dimensional point of view the internal space $\mathcal{M}_{5}$ is a squashed five-dimensional Sasaki-Einstein, as can be seen from (B.3).

Finally, the Einstein eqs. (3.6) reduce to:

$$
R_{\mu \nu}=-\frac{5 \sqrt{5}}{4} W^{2} g_{\mu \nu}
$$

i.e. the external space is $A d S_{5}$ with inverse radius $\lambda$, where:

$$
\lambda^{2}=\frac{5 \sqrt{5}}{16} W^{2} .
$$

This non-supersymmetric $A d S_{5}$ solution was found in [11], and its spectrum was discussed in $[12,13]$. In [14] a truncation of $\mathcal{N}=8$ gauged supergravity to the graviton and four scalars was considered. This truncation admits both the maximally supersymmetric $A d S_{5}$ vacuum and a non-supersymmetric $A d S_{5}$ critical point. The masses of the four scalars at the maximally supersymmetric critical point are such that two of the dual operators are of dimension two, whilst the other two are of dimension three and four. As we will see shortly, the latter two operators are retained in our consistent truncation also. In the non-supersymmetric $A d S_{5}$ critical point of [14], it is the scalar field dual to the operator which is of dimension three in the supersymmetric vacuum which acquires a finite value. In our non-supersymmetric $A d S_{5}$ vacuum, finite values are acquired by both this scalar field and other scalar fields not retained in the truncated action used in [14]. It would be interesting to explore how these critical points are related to each other.

\subsection{Supersymmetric $A d S_{4} \times \mathbb{R} \times \widetilde{S E}_{5}$}

This solution uplifts to the supersymmetric $10 \mathrm{D}$ IIB solution presented in $[9]{ }^{2}$ it can be obtained as a solution of the constrained effective Lagrangian (3.8) by setting:

$$
\alpha=1, \omega=\psi=0 .
$$

The above implies that from the ten-dimensional point of view the internal space $\mathcal{M}_{5}$ is a squashed Sasaki-Einstein, as can be seen from eq. (B.3). Moreover the scalars $\phi, \varphi, \chi$ are assumed to only depend on a single coordinate $r$, and:

$$
\frac{1}{4} \phi^{\prime}=-\frac{\varphi^{\prime}}{2 \varphi}=\frac{\chi^{\prime}}{2 \chi}=\beta
$$

\footnotetext{
${ }^{2}$ Note however that we will only be able to make contact with those solutions of [9] for which $F_{1}=0$.
} 
where the prime denotes differentiation with respect to $r$ and $\beta$ is a constant. In other words the scalars $\phi, \log \varphi, \log \chi$ depend linearly on $r$.

With these substitutions, eqs. (3.3), (3.5) are satisfied, while eq. (3.4) gives:

$$
\beta=\frac{\sqrt{5}}{4} W .
$$

Finally, the Einstein eqs. (3.6) are solved for a geometry of the form $A d S_{4} \times \mathbb{R}$ :

$$
d s^{2}=d s^{2}\left(A d S_{4}\right)+d r^{2} .
$$

More precisely, the components of the Einstein eqs. (3.6) along the $A d S_{4}$ directions reduce to:

$$
R_{\mu \nu}^{(4)}=-3 W^{2} g_{\mu \nu}^{(4)},
$$

which is indeed satisfied by an $A d S_{4}$ space of inverse radius $W$; the $r r$ component of (3.6) is satisfied by virtue of (3.23), while the the mixed components are satisfied automatically.

In [10] $A d S_{D^{-}}$-sliced domain walls were studied within the context of 'fake supergravity', using an adapted superpotential as a useful analysis tool. In order to make contact with their formalism, one must set $\phi=h=0, d=4$ in (2.11) of [10] and moreover identify (there $\rightarrow$ here):

$$
\begin{aligned}
\kappa \phi^{\prime} & \rightarrow \sqrt{\frac{3}{5}} \phi^{\prime} \\
\kappa^{2} V & \rightarrow-\frac{9}{2} W^{2} \\
L_{4}^{2} & \rightarrow \frac{1}{W^{2}} .
\end{aligned}
$$

In particular, the (non-perturbative) stability results established in [10] carry over. Furthermore, the Janus solution of [15] is also a solution of our effective action and thus can be lifted to 10 dimensions using an arbitrary five dimensional manifold admitting a Sasaki-Einstein structure.

\subsection{Non-supersymmetric $A d S_{4} \times \mathbb{R} \times S E_{5}$}

This is the non-supersymmetric solution presented in [10]. This solution was first found in $[16,17]$. It can be obtained as a solution of the constrained effective Lagrangian (3.8) in the limit

$$
\alpha \rightarrow 0
$$

As in the supersymmetric solution the constraint (3.4) is then automatically satisfied, while (3.1) implies:

$$
\varphi=\chi=0
$$

The system (3.5) is then solved for

$$
c_{1} e^{2 \psi}=c_{2} e^{2 \omega}=\frac{1}{\lambda^{2}},
$$


where we have set:

$$
\lambda^{2}:=\left(\frac{5}{6}\right)^{\frac{3}{2}} W^{2} .
$$

As in the supersymmetric case, the equations above imply that the internal space is SasakiEinstein and all fluxes are zero except for the RR five-form:

$$
F_{5}=4 \lambda \operatorname{vol}_{5}
$$

where $\operatorname{vol}_{5}$ denotes the volume form of the internal space. Moreover the scalars $\phi, \varphi, \chi$ are assumed to only depend on a single coordinate $r$, and:

$$
\frac{1}{4} \phi^{\prime}=-\frac{\varphi^{\prime}}{2 \varphi}=\frac{\chi^{\prime}}{2 \chi}=\beta
$$

where the prime denotes differentiation with respect to $r$ and $\beta$ is a constant. In other words the scalars $\phi, \log \varphi, \log \chi$ depend linearly on $r$.

With these substitutions eqs. (3.3), (3.4), (3.5) are satisfied. Moreover, the Einstein eqs. (3.6) are solved for a geometry of the form $A d S_{4} \times \mathbb{R}$ :

$$
d s^{2}=d s^{2}\left(A d S_{4}\right)+d r^{2} .
$$

More precisely, the components of the Einstein eq. (3.6) along the $A d S_{4}$ directions reduce to:

$$
R_{\mu \nu}^{(4)}=-4 \lambda^{2} g_{\mu \nu}^{(4)}
$$

which is indeed satisfied by an $A d S_{4}$ space of inverse square radius $4 \lambda^{2} / 3$; the $r r$ component of (3.6) is satisfied provided:

$$
\beta^{2}=\frac{1}{2} \lambda^{2},
$$

while the mixed components are satisfied automatically.

To translate between [10] and the present, one must set $\phi=h=0, d=4$ in $(2.11)$ of $[10]$ and moreover identify (there $\rightarrow$ here):

$$
\begin{aligned}
\kappa \phi^{\prime} & \rightarrow \frac{1}{\sqrt{2}} \phi^{\prime} \\
\kappa^{2} V & \rightarrow-6 \lambda^{2} \\
L_{4}^{2} & \rightarrow \frac{3}{4 \lambda^{2}} .
\end{aligned}
$$

One can then use the results of this subsection to find a different uplift of the Janus solution. We can also adopt the results of [10] on stability.

\section{Masses}

We will now obtain the masses of the fluctuations around the two critical $A d S_{5} \times \mathcal{M}_{5}$ solutions described earlier. Although the spectra we obtain are valid for general $\mathcal{M}_{5}$, as long as it admits a Sasaki-Einstein structure, in the supersymmetric case of section 4.1 it 
will be useful to specialize to the case where $\mathcal{M}_{5}$ is the five-sphere, in order to identify the dual $\mathrm{CFT}_{4}$ operators using the well-known AdS/CFT dictionary.

In both cases we will take $\lambda$ to be the inverse radius of $A d S_{5}$, i.e:

$$
R=-20 \lambda^{2}
$$

where $R$ is the scalar curvature of $A d S_{5}$. Our conventions are such that for a scalar particle $\sigma$ with equation of motion:

$$
\left(\nabla^{2}-M_{\sigma}^{2}\right) \sigma=0,
$$

the Breitenlohner-Freedman bound in five dimensions reads:

$$
M^{2} \geq-4 \lambda^{2}
$$

4.1 The supersymmetric $A d S_{5} \times \mathcal{M}_{5}$

Let us expand all fields around the critical point as follows:

$$
\begin{aligned}
\phi & =\phi_{0}+\delta \phi \\
e^{2 \psi} & =\frac{1}{c_{1}}\left(\frac{1}{\lambda^{2}}+\delta \psi\right) \\
e^{2 \omega} & =\frac{1}{c_{2}}\left(\frac{1}{\lambda^{2}}+\delta \omega\right) \\
\varphi & =e^{-\phi_{0} / 2} \delta \varphi \\
\chi & =e^{\phi_{0} / 2} \delta \chi,
\end{aligned}
$$

where $\phi_{0}$ is a constant. The inverse radius $\lambda$ of $A d S_{5}$ is related to the constant $W$ through eq. (3.13).

Expanding the equations of motion to first order in the fluctuations we find the following mass spectrum:

\begin{tabular}{|c|c|c|c|c|}
\hline$\sigma$ & $\left(M_{\sigma} / \lambda\right)^{2}$ & $10 \mathrm{D}$ & $\mathcal{O}$ & $\Delta$ \\
\hline$\delta \phi$ & 0 & $B$ & $\operatorname{tr} F_{+}^{2}$ & 4 \\
\hline $4 \delta \psi+\delta \omega$ & 32 & $h_{\alpha}^{\alpha}$ & $\operatorname{tr} F_{+}^{2} F_{-}^{2}$ & 8 \\
\hline$\delta \psi-\delta \omega$ & 12 & $h_{(\alpha \beta)}$ & $\operatorname{tr} \lambda \lambda \bar{\lambda} \bar{\lambda}$ & 6 \\
\hline$\delta \varphi+\delta \chi$ & -3 & $A_{\alpha \beta}$ & $\operatorname{tr} \lambda \lambda$ & 3 \\
\hline$\delta \varphi-\delta \chi$ & 21 & $A_{\alpha \beta}$ & $\operatorname{tr} F_{+}^{2} \bar{\lambda} \bar{\lambda}$ & 7 \\
\hline
\end{tabular}

We see that the dilaton $\phi$ is a flat direction. The negative mass-square is of course above the Breitenlohner-Freedman bound (4.3).

In the third column of the table above we have indicated the ten-dimensional origin of the corresponding perturbation, in the notation of [18]. Namely, $B$ is related to the axiondilaton; $h_{\alpha}^{\alpha}$ and $h_{(\alpha \beta)}$ are the trace and traceless part respectively of the internal-space metric; $A_{\alpha \beta}$ is the internal part of the complex two-form potential. In the fourth column $(\mathcal{O})$ we have listed the dual $\mathrm{CFT}_{4}$ operators, in the notation used in table 7 of [19], with their corresponding dimensions $(\Delta)$ given in the last column. 
The table 7 of [19] also indicates how operators are obtained as supersymmetric descendants of the operators ${ }^{3} \operatorname{tr} X^{k}$. This implies that the spectrum of operators listed above has the following structure:

\begin{tabular}{|c|c|c|}
\hline$\Delta$ & $\mathcal{O}$ & $Q$ descendant \\
\hline 3 & $\mathcal{O}_{3}:=\operatorname{tr} \lambda \lambda$ & $Q^{2}$ \\
\hline 4 & $\mathcal{O}_{4}:=\operatorname{tr} F_{+}^{2}$ & $Q^{4}$ \\
\hline 6 & $\mathcal{O}_{6}:=\operatorname{tr} \lambda \lambda \bar{\lambda} \bar{\lambda}$ & $Q^{2} \bar{Q}^{2}$ \\
\hline 7 & $\mathcal{O}_{7}:=\operatorname{tr} F_{+}^{2} \bar{\lambda} \bar{\lambda}$ & $Q^{4} \bar{Q}^{2}$ \\
\hline 8 & $\mathcal{O}_{8}:=\operatorname{tr} F_{+}^{2} F_{-}^{2}$ & $Q^{4} \bar{Q}^{4}$ \\
\hline
\end{tabular}

From this table we see that the only non-vanishing terms in the operator product expansions are:

$$
\mathcal{O}_{3} \mathcal{O}_{6} \sim \mathcal{O}_{7} ; \quad \mathcal{O}_{3} \mathcal{O}_{3} \sim \mathcal{O}_{4} ; \quad \mathcal{O}_{6} \mathcal{O}_{6} \sim \mathcal{O}_{8}
$$

with all other products vanishing because they contain more than four $Q$ or $\bar{Q}$. This argument from the field theory justifies why this set of five operators has a closed OPE, and thus explains why there is a consistent supergravity truncation to these modes.

In [20] a related consistent truncation of supergravity was obtained which contains three additional scalar fields. The additional modes are dual to operators $\mathcal{O}_{3}^{\prime}:=\operatorname{tr} \bar{\lambda} \bar{\lambda} \sim \bar{Q}^{2}$; $\mathcal{O}_{4}^{\prime}:=\operatorname{tr} F_{-}^{2} \sim \bar{Q}^{4}$ and $\mathcal{O}_{7}^{\prime}:=\operatorname{tr} F_{-}^{2} \bar{\lambda} \bar{\lambda} \sim Q^{2} \bar{Q}^{4}$. These three operators have a trivial OPE with each other, but together with the other five operators generate a closed OPE in which:

$$
\begin{aligned}
\mathcal{O}_{3}^{\prime} \mathcal{O}_{3} \sim \mathcal{O}_{6} ; & \mathcal{O}_{3}^{\prime} \mathcal{O}_{4} \sim \mathcal{O}_{7} ; & \mathcal{O}_{3}^{\prime} \mathcal{O}_{6} \sim \mathcal{O}_{7}^{\prime} ; & \mathcal{O}_{3}^{\prime} \mathcal{O}_{7} \sim \mathcal{O}_{8} \\
\mathcal{O}_{4}^{\prime} \mathcal{O}_{3} \sim \mathcal{O}_{7}^{\prime} ; & \mathcal{O}_{4}^{\prime} \mathcal{O}_{4} \sim \mathcal{O}_{8} ; & \mathcal{O}_{7}^{\prime} \mathcal{O}_{3} \sim \mathcal{O}_{8} &
\end{aligned}
$$

The existence of this closed OPE of eight operators thus explains the origin of the consistent supergravity truncation in this case also.

\subsection{The non-supersymmetric $A d S_{5} \times \mathcal{M}_{5}$}

We expand the fields around the critical point as follows:

$$
\begin{aligned}
\phi & =\phi_{0}+\delta \phi \\
e^{2 \psi} & =\frac{2}{\sqrt{5}}(1+\delta \psi) \\
e^{2 \omega} & =\frac{\sqrt{5}}{2}(1+\delta \omega) \\
\varphi & =\sqrt{2} e^{-\phi_{0} / 2}(1+\delta \varphi) \\
\chi & =\sqrt{2} e^{\phi_{0} / 2}(1+\delta \chi),
\end{aligned}
$$

where $\phi_{0}$ is a constant. The inverse radius $\lambda$ of $A d S_{5}$ is related to the constant $W$ through eq. (3.20).

\footnotetext{
${ }^{3}$ Strictly speaking, our case is the degenerate case, $k=0$.
} 
Expanding the equations of motion to first order in the fluctuations we find the following mass spectrum:

\begin{tabular}{|c|c|c|}
\hline$\sigma$ & $\left(M_{\sigma} / \lambda\right)^{2}$ & $\Delta$ \\
\hline $2 \delta \phi-\delta \varphi+\delta \chi$ & 0 & 4 \\
\hline$-\delta \phi-\delta \varphi+\delta \chi$ & 24 & $2(1+\sqrt{7})$ \\
\hline$\delta \psi+\delta \omega$ & 32 & 8 \\
\hline $4 \delta \psi+\delta \varphi+\delta \chi$ & 32 & 8 \\
\hline$-2 \delta \psi+\delta \varphi+\delta \chi$ & 8 & $2(1+\sqrt{3})$ \\
\hline
\end{tabular}

As in the supersymmetric solution, there is again a single flat direction and all masssquares are positive. Note that an operator of dimension $2(1+\sqrt{3})$ was also found in the analysis of the spectrum around the non-supersymmetric $A d S_{5}$ vacuum in $[12,13]$.

\section{Conclusions}

One of the motivations for this work was to develop holography for $A d S_{4} \times \mathbb{R} \times \mathcal{M}_{5}$ IIB solutions. In this paper we have shown that both supersymmetric and non-supersymmetric solutions can be obtained as solutions of five-dimensional consistent truncations. As a next step one would like to use the truncated action as a starting point for setting up a holographic dictionary. Recall that in the familiar case of $A d S_{5}$, the consistent truncation to five-dimensional Einstein gravity with cosmological constant captures only the stress energy tensor of the dual conformal theory. By considering asymptotically locally $A d S_{5}$ solutions of the form

$$
d s^{2}=\frac{d \rho^{2}}{4 \rho^{2}}+\frac{1}{\rho}\left(g_{(0) i j} d x^{i} d x^{j}+\rho g_{(2) i j}+\rho^{2} g_{(4) i j} \cdots\right) d x^{i} d x^{j},
$$

near the AdS conformal boundary as $\rho \rightarrow 0$, in which $g_{(0) i j}$ acts as the source for the CFT stress energy tensor, we set up a holographic dictionary by analyzing such geometries and computing renormalized correlators of the CFT stress energy tensor, see [21, 22]. An important step in this analysis is to generalize the $A d S_{5}$ solution to solutions which asymptotically locally approach $A d S_{5}$.

Returning now to the case of $A d S_{4} \times \mathbb{R}$, the key issue is to establish the structure of the conformal boundary. Note that since the coordinate along $\mathbb{R}$ is non-compact one cannot dimensionally reduce to four dimensions; more precisely, the conformal boundary of the five-dimensional spacetime is not the conformal boundary of $A d S_{4}$. A related discussion appeared in the context of the Janus solution. These solutions are AdS sliced domain walls, in which the conformal boundary consists of two half-spheres with angular excess joined at the equator, and the coupling constant (dilaton) takes different values on either side; see for example section 4.1 of [23]. In fact the non-supersymmetric $A d S_{4} \times \mathbb{R}$ can be obtained as a particular limit of the Janus solution; however, the analysis of [23] degenerates in this limit.

One natural idea is to change the conformal frame in five dimensions to obtain a metric which is asymptotically locally $A d S_{5}$. This idea was previously applied to non-conformal 
branes (i.e. running dilaton backgrounds, see [24]). The non-conformal brane geometries admit a distinguished conformal frame in which the geometry becomes AdS with the dilaton running; the geometries capture the dual theory in the region where the dynamics is driven by the dimensionality of the coupling constant, see for example section 3 of [24]. The $A d S_{4} \times \mathbb{R}$ solutions also become asymptotically locally AdS by a change of frame and it would be interesting to exploit this fact in order to set up the holographic dictionary for this class of solutions. We hope to return to this issue in the future.

It seems possible that the supersymmetric Janus solutions of [25] may also be recovered within the framework of our effective action. The authors of that reference constructed their solutions using (a deformation of) $S^{5}$ as their internal space. Since our reduction ansatz makes no reference to any specific $\mathcal{M}_{5}$, as long as it admits a Sasaki-Einstein structure, if the Janus solutions can indeed be obtained using our effective action this would demonstrate that they admit straightforward generalizations to arbitrary five-manifolds admitting Sasaki-Einstein structure. It would be interesting to explore this further.

Note added. While this paper was being prepared, we received preprint [20] which has significant overlap with our results. In particular, it should be possible to show that the five-dimensional effective action (2.8) of the present paper is a truncation to the graviton coupled to five real scalars of the supersymmetric five-dimensional effective action presented in [20]. The mass fluctuations around the two $A d S_{5}$ vacua presented in our section 4 can also be identified with the corresponding mass spectra presented in tables (5.6) and (5.16) of $[20] .{ }^{4}$

\section{Acknowledgments}

M.T. and D.T. would like to thank the organizers of the 5th Regional Meeting in String Theory in Kolymbari, Crete, where this work was initiated. This work is part of the research program of the Stichting voor Fundamenteel Onderzoek der Materie (FOM), which is financially supported by the Nederlandse Organisatie voor Wetenschappelijk Onderzoek (NWO). Two of the authors acknowledge support from NWO: K.S. via a Vici grant, M.T. via the Vidi grant 'Holography, duality and time dependence in string theory'.

\section{A Five-dimensional Sasaki-Einstein manifolds}

As already mentioned in section 2 , the internal five-dimensional manifold $\mathcal{M}_{5}$ of our tendimensional reduction ansatz can be obtained from a Sasaki-Einstein manifold through warping and squashing. Furthermore, associated with the Sasaki-Einstein structure there is an underlying $\mathrm{SU}(2)$ structure which was crucial for obtaining the supersymmetric solutions of [9], and is also central to the consistent truncation of the present paper. In this

\footnotetext{
${ }^{4}$ Note, however, that there is an overall factor of $8 / 9$ discrepancy in the mass spectra around the nonsupersymmetric $A d S_{5}$ vacuum between our results and those of [20]; our masses are normalized with respect to the inverse radius of the corresponding $A d S_{5}$ space. Moreover our results suggest that there is a typo in the fifth line of table (5.16) of [20] and one should replace $\left(\operatorname{Im} \delta b^{\Omega}, \operatorname{Re} \delta c^{\Omega}\right)$ with $\left(\operatorname{Re} \delta b^{\Omega}, \operatorname{Im} \delta c^{\Omega}\right)$.
} 
appendix we explain some of the relevant geometrical concepts. For further details the reader may consult [9].

In five dimensions a Sasaki-Einstein manifold may be defined under certain additional mild assumptions as one which admits a pair of Killing spinors (related to each other by complex conjugation). From that it follows that the metric is Einstein. With the canonical normalization of a 'unit' Sasaki-Einstein space in five dimensions, the Ricci tensor of $\mathcal{M}_{5}$ is given by:

$$
R_{m n}^{(S E)}=4 g_{m n}^{(S E)}
$$

so that the six-dimensional cone $\mathcal{C}\left(\mathcal{M}_{5}\right)$ is Calabi-Yau.

Moreover it can be shown that one can construct three real two-forms $\alpha, \beta, \gamma$ and a real one-form $u$, as bilinears of the Killing spinor, obeying the algebraic conditions:

$$
\begin{gathered}
\iota_{u} \alpha=\iota_{u} \beta=\iota_{u} \gamma=0 \\
\alpha \wedge \beta=\beta \wedge \gamma=\gamma \wedge \alpha=0 \\
\alpha \wedge \alpha=\beta \wedge \beta=\gamma \wedge \gamma \neq 0 .
\end{gathered}
$$

Therefore, the forms $(\alpha, \beta, \gamma, u)$ define an associated local $\mathrm{SU}(2)$ structure. In addition the Killing spinor property can be used to show that the following differential conditions are obeyed:

$$
\mathrm{d} u=-2 \gamma ; \quad \mathrm{d}(\alpha+i \beta)=-3 i u \wedge(\alpha+i \beta) ; \quad \mathrm{d} \gamma=0 .
$$

The Sasaki-Einstein metric associated with the SU(2) structure (A.2), (A.3) can locally be put in the canonical form:

$$
d s_{S E}^{2}=d s_{K E}^{2}+u \otimes u,
$$

where $d s_{K E}^{2}$ is a Kähler-Einstein four-dimensional base over which $u$ is fibered. The connection field strength of this local $\Upsilon(1)$ fibration is the Kähler form of the base, and is equal to $d u=-2 \gamma$. If in addition the orbits of the vector dual to $u$ (which is known as the 'Reeb vector') are closed and the associated $\Upsilon(1)$ action is free, (A.4) extends globally and the base is a four-dimensional Kähler-Einstein manifold of positive curvature.

In appendix $\mathrm{B}$ we explain in detail how the local $\mathrm{SU}(2)$ structure $(\alpha, \beta, \gamma, u)$ can be used to build the reduction ansatz leading to the consistent truncation.

\section{B Consistent truncation}

In this appendix we give some technical details concerning the consistent truncation presented in section 2 .

Our starting point is the ten-dimensional type IIB supergravity in the string frame. The equations of motion can be derived from the following pseudoaction:

$$
S=\frac{1}{2 \kappa_{10}^{2}} \int \mathrm{d}^{10} x \sqrt{-g}\left\{e^{-2 \phi}\left[R+4(\partial \phi)^{2}-\frac{1}{2} H^{2}\right]-\frac{1}{4} F^{2}\right\},
$$

where we are using polyform notation. Our choice of notation and conventions follows appendix A of [28], which the reader may consult for further details. 
Let us now describe the reduction ansatz. For the 10D metric in the string frame we set:

$$
d s_{10}^{2}=e^{2 A(x)}\left\{g_{\mu \nu} d x^{\mu} d x^{\nu}+d s^{2}\left(\mathcal{M}_{5}\right)\right\},
$$

where $(\mu, \nu)$ are indices along the $5 \mathrm{D}$ spacetime, and $\mathcal{M}_{5}$ is the five-dimensional internal manifold. Furthermore we shall take the internal metric to be of the form:

$$
d s^{2}\left(\mathcal{M}_{5}\right)=c_{1} e^{2 \psi(x)} d s_{K E}^{2}+c_{2} e^{2 \omega(x)} u \otimes u,
$$

where

$$
d s_{K E}^{2}+u \otimes u,
$$

is the metric of a canonically normalized 'unit' five-dimensional Sasaki-Einstein space (cf. appendix A). All three functions $A, \psi, \omega$ are assumed to depend only on the 5D spacetime coordinates $x^{\mu}$. The constants $c_{1}, c_{2}$ in (B.3) are chosen as follows:

$$
c_{1}=\frac{6}{5 W^{2}}, \quad c_{2}=\left(\frac{6}{5 W}\right)^{2},
$$

so that in the $\psi=\omega=0$ limit the internal metric (B.3) reduces to the squashed SasakiEinstein internal space of the type IIB $\mathcal{N}=1$ solution of [9]. $W$ is an arbitrary real constant within supergravity, however upon imposing flux quantization it will be constrained to discrete values.

Without loss of generality we can take the dilaton to be given by:

$$
\phi=4 A,
$$

so that the 10D Einstein-frame metric (2.1) is obtained from (B.2) by simply dropping the overall warping.

The ansatz for the NSNS three-form flux is given by:

$$
H=\frac{1}{4 f} W \operatorname{Re} \Omega \wedge d \chi-2 \sqrt{c_{2}} f \chi J \wedge u,
$$

where $\chi(x)$ is a $5 \mathrm{D}$ spacetime scalar, and we have set:

$$
f:=\frac{\sqrt{5}}{4} W,
$$

for later convenience; the two-forms $J, \Omega$ are related to the underlying $\mathrm{SU}(2)$ structure of $\mathcal{M}_{5}$ via:

$$
\begin{aligned}
J & =c_{1}(\sin \theta \alpha+\cos \theta \beta) \\
\Omega & =c_{1}(\cos \theta \alpha-\sin \theta \beta-i \gamma),
\end{aligned}
$$

where $\theta$ is constant. The real two-forms $\alpha, \beta, \gamma$ and the real one-form $u$ define a local SU(2) structure on the internal space, obeying the algebraic and differential conditions given in eqs. (A.2), (A.3). 
The RR fluxes are given by:

$$
\begin{aligned}
e^{\phi} F_{1} & =0 \\
e^{\phi} F_{3} & =\frac{1}{4 f} W J \wedge d \varphi+2 \sqrt{c_{2}} f \varphi \operatorname{Re} \Omega \wedge u \\
e^{\phi} F_{5} & =\left(2-\frac{1}{2} \chi \varphi\right) \sqrt{c_{2}} W J \wedge J \wedge u .
\end{aligned}
$$

The total five-form flux is self-dual and given by $F_{5}^{\text {tot }}=F_{5}+\star_{10} F_{5}$.

The above ansatz guarantees that the fluxes obey the Bianchi identities:

$$
d H=0, \quad d F+H \wedge F=0,
$$

where we are using polyform notation.

The combination $R_{M N}^{(10)}+2 \nabla_{M}^{(10)} \partial_{N} \phi$, where $R_{M N}^{(10)}$ is the Ricci tensor and $\nabla_{M}^{(10)}$ the covariant derivative associated with the metric in (B.2), appears in the ten-dimensional stringframe Einstein equations; $(M, N)$ are ten-dimensional Einstein indices. Taking (B.2), (B.6) into account, we compute:

$$
\begin{aligned}
\left(R^{(10)}+2 \nabla^{(10)} \partial \phi\right)_{\mu n}= & 0 \\
\left(R^{(10)}+2 \nabla^{(10)} \partial \phi\right)_{\mu \nu}= & R_{\mu \nu}^{(5)}-g_{\mu \nu}\left(\nabla^{2} A+\partial(4 \psi+\omega) \cdot \partial A\right) \\
& -8 \partial_{\mu} A \partial_{\nu} A-4 \partial_{\mu} \psi \partial_{\nu} \psi-\partial_{\mu} \omega \partial_{\nu} \omega-\nabla_{\mu} \partial_{\nu}(4 \psi+\omega) \\
\left(R^{(10)}+2 \nabla^{(10)} \partial \phi\right)_{m n}= & -u_{m} u_{n} c_{2} e^{2 \omega}\left\{\nabla^{2}(A+\omega)+\partial(4 \psi+\omega) \cdot \partial(A+\omega)-\frac{4 c_{2} e^{2 \omega}}{c_{1}^{2} e^{4 \psi}}\right\} \\
& -\tilde{g}_{m n}\left\{\nabla^{2}(A+\psi)+\partial(4 \psi+\omega) \cdot \partial(A+\psi)-\frac{6}{c_{1} e^{2 \psi}}\left(1-\frac{c_{2} e^{2 \omega}}{3 c_{1} e^{2 \psi}}\right)\right\},
\end{aligned}
$$

where the metric $\tilde{g}_{m n}$ is given by:

$$
\tilde{g}_{m n} d x^{m} \otimes d x^{n}=c_{1} e^{2 \psi} d s_{K E}^{2} .
$$

In addition to the above, in order to reduce the 10D equations of motion using our ansatz, one needs to make use of the identities obeyed by the $\mathrm{SU}(2)$ structure listed in [26, 27]. Taking into account all of the above, we finally obtain the following:

- The 10D dilaton eom reduces to

$$
\begin{aligned}
\nabla^{\mu}\left(e^{4 \psi+\omega} \partial_{\mu} A\right)= & \frac{W^{2}}{64 f^{2}}\left\{e^{4 A+\omega}(\partial \varphi)^{2}-e^{-4 A+\omega}(\partial \chi)^{2}\right\} \\
& +f^{2}\left(e^{4 A-\omega} \varphi^{2}-e^{-4 A-\omega} \chi^{2}\right) .
\end{aligned}
$$

- The internal piece of the 10D Einstein eom reduces to two scalar equations

$$
\begin{aligned}
\nabla^{\mu}\left(e^{4 \psi+\omega} \partial_{\mu} \psi\right)= & -\frac{W^{2}}{64 f^{2}}\left\{e^{4 A+\omega}(\partial \varphi)^{2}+e^{-4 A+\omega}(\partial \chi)^{2}\right\} \\
& -W^{2}\left(2-\frac{1}{2} \chi \varphi\right)^{2} e^{-4 \psi-\omega}+\frac{6}{c_{1}}\left(1-\frac{c_{2} e^{2 \omega}}{3 c_{1} e^{2 \psi}}\right) e^{2 \psi+\omega} \\
& -f^{2}\left(e^{4 A-\omega} \varphi^{2}+e^{-4 A-\omega} \chi^{2}\right)
\end{aligned}
$$


and

$$
\begin{aligned}
\nabla^{\mu}\left(e^{4 \psi+\omega} \partial_{\mu} \omega\right)= & \frac{W^{2}}{64 f^{2}}\left\{e^{4 A+\omega}(\partial \varphi)^{2}+e^{-4 A+\omega}(\partial \chi)^{2}\right\} \\
& -W^{2}\left(2-\frac{1}{2} \chi \varphi\right)^{2} e^{-4 \psi-\omega}+\frac{4 c_{2}}{c_{1}^{2}} e^{3 \omega} \\
& -3 f^{2}\left(e^{4 A-\omega} \varphi^{2}+e^{-4 A-\omega} \chi^{2}\right) .
\end{aligned}
$$

- The mixed piece of the 10D Einstein eom is automatically satisfied.

- The external piece of the $10 \mathrm{D}$ Einstein eom reduces to

$$
\begin{aligned}
0= & e^{4 \psi+\omega} R_{\mu \nu}^{(5)}+e^{4 \psi+\omega}\left(12 \partial_{\mu} \psi \partial_{\nu} \psi+8 \partial_{\mu} \psi \partial_{\nu} \omega-8 \partial_{\mu} A \partial_{\nu} A\right) \\
& -\frac{W^{2}}{16 f^{2}}\left(e^{4 A+\omega} \partial_{\mu} \varphi \partial_{\nu} \varphi+e^{-4 A+\omega} \partial_{\mu} \chi \partial_{\nu} \chi\right)-\nabla_{\mu} \partial_{\nu} e^{4 \psi+\omega} \\
& -\frac{1}{3} g_{\mu \nu}\left\{\nabla^{2} e^{4 \psi+\omega}+2 W^{2}\left(2-\frac{1}{2} \chi \varphi\right)^{2} e^{-4 \psi-\omega}\right. \\
& \left.-\frac{24}{c_{1}}\left(1-\frac{c_{2} e^{2 \omega}}{6 c_{1} e^{2 \psi}}\right) e^{2 \psi+\omega}+4 f^{2}\left(e^{4 A-\omega} \varphi^{2}+e^{-4 A-\omega} \chi^{2}\right)\right\},
\end{aligned}
$$

where we have taken (B.14) into account.

- The $H$-form eom reduces to

$$
\nabla^{\mu}\left(e^{-4 A+\omega} \partial_{\mu} \chi\right)=20 f^{2} e^{-4 A-\omega} \chi-16 f^{2} \varphi\left(2-\frac{1}{2} \chi \varphi\right) e^{-4 \psi-\omega} .
$$

- The $F_{1}$-form eom is automatically satisfied.

- The $F_{3}$-form eom reduces to

$$
\nabla^{\mu}\left(e^{4 A+\omega} \partial_{\mu} \varphi\right)=20 f^{2} e^{4 A-\omega} \varphi-16 f^{2} \chi\left(2-\frac{1}{2} \chi \varphi\right) e^{-4 \psi-\omega} .
$$

- The $F_{5}$-form eom is automatically satisfied.

It is now straightforward to see that equations (B.14-B.19) can be 'integrated' to the five-dimensional effective action given in eq. (2.8) of section 2. As might have been expected, the Lagrangian (2.8) turns out to be equivalent to the string-frame ten-dimensional IIB pseudoaction in eq. (B.1) upon substitution of the ansatz (B.2), (B.6), (B.7), (B.10) and integration over the internal directions.

Open Access. This article is distributed under the terms of the Creative Commons Attribution Noncommercial License which permits any noncommercial use, distribution, and reproduction in any medium, provided the original author(s) and source are credited. 


\section{References}

[1] K. Skenderis and M. Taylor, Kaluza-Klein holography, JHEP 05 (2006) 057 [hep-th/0603016] [SPIRES].

[2] B. de Wit and H. Nicolai, The Consistency of the $S^{7}$ Truncation in $D=11$ Supergravity, Nucl. Phys. B 281 (1987) 211 [SPIRES].

[3] H. Nastase, D. Vaman and P. van Nieuwenhuizen, Consistent nonlinear $K K$ reduction of 1 $1 D$ supergravity on $A d S_{7} \times S^{4}$ and self-duality in odd dimensions, Phys. Lett. B 469 (1999) 96 [hep-th/9905075] [SPIRES].

[4] H. Nastase, D. Vaman and P. van Nieuwenhuizen, Consistency of the AdS $\times S^{4}$ reduction and the origin of self-duality in odd dimensions, Nucl. Phys. B 581 (2000) 179 [hep-th/9911238] [SPIRES].

[5] H. Lü, C.N. Pope and T.A. Tran, Five-dimensional $N=4, \mathrm{SU}(2) \times \Upsilon(1)$ gauged supergravity from type IIB, Phys. Lett. B 475 (2000) 261 [hep-th/9909203] [SPIRES].

[6] M. Cvetič, H. Lü, C.N. Pope, A. Sadrzadeh and T.A. Tran, Consistent SO(6) reduction of type IIB supergravity on $S^{5}$, Nucl. Phys. B 586 (2000) 275 [hep-th/0003103] [SPIRES].

[7] J. Maldacena, D. Martelli and Y. Tachikawa, Comments on string theory backgrounds with non-relativistic conformal symmetry, JHEP 10 (2008) 072 [arXiv:0807.1100] [SPIRES].

[8] J.P. Gauntlett and O. Varela, Consistent Kaluza-Klein Reductions for General Supersymmetric AdS Solutions, Phys. Rev. D 76 (2007) 126007 [arXiv:0707.2315] [SPIRES].

[9] D. Lüst and D. Tsimpis, New supersymmetric AdS 4 type-II vacua, JHEP 09 (2009) 098 [arXiv:0906.2561] [SPIRES].

[10] D.Z. Freedman, C. Núñez, M. Schnabl and K. Skenderis, Fake Supergravity and Domain Wall Stability, Phys. Rev. D 69 (2004) 104027 [hep-th/0312055] [SPIRES].

[11] L.J. Romans, New compactifications of chiral $N=2 D=10$ supergravity, Phys. Lett. B 153 (1985) 392 [SPIRES].

[12] J. Distler and F. Zamora, Non-supersymmetric conformal field theories from stable anti-de Sitter spaces, Adv. Theor. Math. Phys. 2 (1999) 1405 [hep-th/9810206] [SPIRES].

[13] J. Distler and F. Zamora, Chiral symmetry breaking in the AdS/CFT correspondence, JHEP 05 (2000) 005 [hep-th/9911040] [SPIRES].

[14] D.Z. Freedman, S.S. Gubser, K. Pilch and N.P. Warner, Renormalization group flows from holography supersymmetry and a c-theorem, Adv. Theor. Math. Phys. 3 (1999) 363 [hep-th/9904017] [SPIRES].

[15] D. Bak, M. Gutperle and S. Hirano, A dilatonic deformation of $A d S_{5}$ and its field theory dual, JHEP 05 (2003) 072 [hep-th/0304129] [SPIRES].

[16] T. Robb and J.G. Taylor, Ade $S \times S^{1} \times M_{5}$ compact solutions for $N=2 D=10$ chiral supergravity, Phys. Lett. B 155 (1985) 59 [SPIRES].

[17] F. Quevedo, Compactification of chiral $N=2 D=10$ supergravity, Phys. Lett. B 173 (1986) 145 [SPIRES].

[18] H.J. Kim, L.J. Romans and P. van Nieuwenhuizen, The Mass Spectrum of Chiral $N=2$ $D=10$ Supergravity on $S^{5}$, Phys. Rev. D 32 (1985) 389 [SPIRES]. 
[19] E. D'Hoker and D.Z. Freedman, Supersymmetric gauge theories and the AdS/CFT correspondence, hep-th/0201253 [SPIRES].

[20] D. Cassani, G. Dall'Agata and A.F. Faedo, Type IIB supergravity on squashed Sasaki-Einstein manifolds, arXiv:1003.4283 [SPIRES].

[21] S. de Haro, S.N. Solodukhin and K. Skenderis, Holographic reconstruction of spacetime and renormalization in the AdS/CFT correspondence, Commun. Math. Phys. 217 (2001) 595 [hep-th/0002230] [SPIRES].

[22] K. Skenderis, Lecture notes on holographic renormalization, Class. Quant. Grav. 19 (2002) 5849 [hep-th/0209067] [SPIRES].

[23] I. Papadimitriou and K. Skenderis, Correlation functions in holographic RG flows, JHEP 10 (2004) 075 [hep-th/0407071] [SPIRES].

[24] I. Kanitscheider, K. Skenderis and M. Taylor, Precision holography for non-conformal branes, JHEP 09 (2008) 094 [arXiv:0807.3324] [SPIRES].

[25] E. D'Hoker, J. Estes and M. Gutperle, Ten-dimensional supersymmetric Janus solutions, Nucl. Phys. B 757 (2006) 79 [hep-th/0603012] [SPIRES].

[26] D. Lüst and D. Tsimpis, Classes of AdS 4 type IIA/IIB compactifications with $\mathrm{SU}(3) \times \mathrm{SU}(3)$ structure, JHEP 04 (2009) 111 [arXiv: 0901.4474] [SPIRES].

[27] J. Bovy, D. Lüst and D. Tsimpis, $N=1,2$ supersymmetric vacua of IIA supergravity and $\mathrm{SU}(2)$ structures, JHEP 08 (2005) 056 [hep-th/0506160] [SPIRES].

[28] D. Lüst, F. Marchesano, L. Martucci and D. Tsimpis, Generalized non-supersymmetric flux vacua, JHEP 11 (2008) 021 [arXiv:0807.4540] [SPIRES]. 